\title{
Applications of Accelerators
}

\section{Rosen}

It is important to step back, from time to time, and look at our work in the context of the preoccupations of society as a whole. This article takes a look at what role accelerators are playing in modern life.

We could say that science should not need technology as a raison d'être but there is no doubt that, when science is costing a lot of money, to be able to point to subsequent technological applications is an important influence in encouraging continued investment in science. When, in the process of increasing our knowledge of man's environment, we develop the technology which eases man's lot in his environment, it is a bonus we should be very ready to talk about. Thinking of accelerators, the financial benefits already drown the iinancial investments as this article brings out.

The author (Louis Rosen - Director of the Los Alamos Meson Physics Facility) always presents our work, and LAMPF in particular, is a broad context. In this article he concentrates on what has come, and is coming. from our mastery of acceleration techniques. It is taken from a talk 'Relevance of Particle Accelerators to National Goals' given at the 1971 Particle Accelerator Conference held in Chicago in March. This is a version of the talk, abridged and generalized, to be accessible to a wider audience, by kind permission of the author.
There was a time, not long ago, when science and motherhood were beyond reproach. Today, both are under attack.

Much of the basis for the attack on science is emotional, even irrational. But not all of our troubles can be blamed on unreasoning critics; a substantial part of our misery is selfinflicted. We have not taken seriously that part of our responsibility to society which dictates that we explain, interpret and justify our activities in language understandable to the nonspecialist. We and we alone can do that. We and we alone can provide the best advice on meaningful priorities based on the intellectual and practical worth of our pursuits. We and we alone can provide the best assessment that a given development will have a. utilitarian purpose at an acceptable cost. We and we alone can develop the sophisticated phenomenological models which have some chance of predicting the interactions between technology, industry, education, society and our environment.

We are admonished from many quarters to start asking not what our society can do for science, but what science can do for our society. And it is precisely this question, to the extent it concerns particle accelerators, that I wish to discuss.

If we look at the world-wide inventory of particle accelerators, we could claim that they all have value for intellectual and educational pursuits. However, most of us feel that basic knowledge about the constituents of matter and about the forces that govern the most fundamental properties of sub-nuclear matter are most likely to arise from experiments at the highest energies, assuming that sufficient intensity is available to make statistically significant observations. It is the highest energy accelerators, particularly, that contribute to education and the acquisition of new basic knowledge.

To understand the importance of these contributions, we must recognize that one of the main distinguishing characteristics between man and the lower forms of animal life is his curiosity - curiosity about himself, his immediate surroundings and the universe. Curiosity is one of the elements of life which gives it substance and meaning, and one of the major ways to satisfy human curiosity is through the pursuit of science - the interrogation of nature. In order to pursue science, one must continually press on the frontiers which are usually at extremes: very high temperatures and very low temperatures; very high pressures and very good vacuums; the very large (cosmology) and the very small (nuclear and sub-nuclear entities). High energy particle accelerators permit us to explore the smallest quantities of matter and energy in nature.

In addition to these intellectual merits, we can point to other benefits from the construction and utilization of accelerators. For example, one that we will pass over briefly, is the promotion of international collaboration. The research is world-wide and, perhaps, in no other field is there such open, friendly and practical collaboration across frontiers.

Let us push on further and get to some directly demonstrable applications of accelerators. The history of science tells us that, up to now, the practical results alone have more than paid for all the scientific effort. Even the highest energy accelerators already have economic ramifications for they are producing technological spin-offs (for example in computer technology, cryogenics, vacuum technology, the art of constructing large magnetic fields, and of fabricating 
A list of some of the applications of accelerators with numbers assigned to each category and the capital investment involved (taken from a report of the Subpanel on Accelerators to the Nuclear Science Panel of the Physics Survey of the National Academy of Sciences, USA). materials which have no electrical resistance) all of which will have a decisive influence on the technologies required to sustain comfortable life on this planet in the future.

But let us examine not what might be but what already is, remembering that what today are considered low energy accelerators were yesterday characterized as high energy accelerators.

\section{Accelerators in industry}

If we look at the situation in the United States there are about 1000 accelerators of all kinds, representing about $50 \%$ of the world's inventory of accelerators. Less than 150 are devoted mainly to basic research. Of the remainder, about one-third are devoted to industry and medicine, and the rest to the applied sciences. Those devoted to industry and medicine represent a capital investment of 77 million dollars. The annual production of goods and services associated with these machines is about 2000 million dollars.

Non destructive testing

A growing use of accelerators is in the area of nondestructive testing. There are three main categories:

1) Radiographic inspection using $x$ rays and gamma rays (e.g., inspection of pipeline welds)

2) Thickness gauges (alphas and betas have long been used for this purpose and now protons are beginning to show promise using $147 \mathrm{MeV}$ protons, the Harwell Group have shown that the thickness of graphite can be determined to an accuracy of $0.0015 \%$, compared to $2 \%$ by conventional methods)

3) Activation analysis (mainly with neutrons).

Radioisotope production

Two-thirds of all radioactive nuclei were discovered via acceleratorinduced reactions. However, $80 \%$ of

\begin{tabular}{|l|c|c|c|c|}
\hline \multicolumn{1}{|c|}{ Application } & \multicolumn{2}{|c|}{$\begin{array}{c}\text { Number of } \\
\text { Accelerators }\end{array}$} & \multicolumn{2}{c|}{ Investment (\$ M) } \\
\hline & 1964 & 1968 & 1964 & 1968 \\
\hline $\begin{array}{l}\text { Nuclear science and } \\
\text { engineering }\end{array}$ & 282 & 297 & -101.4 & 129.7 \\
X rays and neutrons & 234 & 376 & 24.2 & 46.9 \\
Radiation effects & 225 & 315 & 26.3 & 36.4 \\
Atomic and solid state physics & 5 & 35 & 0.5 & 2.8 \\
Radiation processing & 36 & 60 & 3.7 & 6.5 \\
\hline
\end{tabular}

the curies are now produced by reactors. This situation appears to be changing, especially in the medical area to which we shall return.

Market statistics and predictions (in $\$ M)$ for the sale of radioisotopes are as follows :

$\begin{array}{lccr} & 1969 & 1970 & 1971 \\ \text { Basic radionuclides } & 10 & 11 & 13 \\ \text { Radiochemicals } & 12 & 14 & 16 \\ \text { Radiopharmaceuticals } & 32 & 40 & 50 \\ \text { Sealed sources } & 5 & 6 & 7 \\ & \frac{-}{59} & 71 & \frac{76}{}\end{array}$

The point is that the sales are substantial and the rate of increase is large. The present market for cyclotron-produced isotopes is three million dollars per year, and increasing rapidly. It is estimated that a market for about twenty cyclotron facilities may develop for radioisotopes by 1975. Here the economies are far less important than the pain and suffering these isotopes can prevent. Power production

Accelerators have played and continue to play a critical role in development of power sources based on nuclear fuels. This goes to the heart of problems of the conservation of fossil fuels, environmental pollution and the quality of life. Here are a few examples of how nuclear crosssection measurements have contributed :

1) Careful measurements of the ratio of neutron capture to fission for
${ }^{239} \mathrm{Pu}$ showed that an entire family of water-cooled plutonium-fueled reactors would not be feasible as breeder reactors, thus preventing the waste of hundreds of millions of dollars.

2) Some years ago, I published results on the interaction of fast neutrons with ${ }^{7} \mathrm{Li}$, which showed that a controlled thermonuclear reactor could operate on the D-T cycle (which is much easier than the $D-D$ cycle because the required temperature is lower) and produce more tritium than is consumed. It now appears likely that the first thermonuclear reactors will operate on the D-T cycle.

However, Rand McNally Jr. of ORNL has recently proposed the use of energetic protons, (or deuterons, tritons and ${ }^{3} \mathrm{He}$ ) to ignite ${ }^{6} \mathrm{Li}$ or ${ }^{6} \mathrm{LiD}$ fuel, thus avoiding the problem of heating incoming fuel material to fusion energies. One barrier to the pursuit of this idea is grossly incomplete knowledge of nuclear reaction cross-sections for light nuclei at low energies, which accelerators can provide.

It is a fact that particle accelerators provide the basic information for calculating nuclear properties of reactors. Much basic nuclear data are still needed, especially for fast reactors which make best use of our uranium resources. Annual fuel cost 
uncertainties, resulting from nuclear data uncertainties, are about $\$ 100$ million in 1980, \$300 million in 1990 , and $\$ 700$ million by the end of the century. Accelerators can clear these uncertainties.

Neutron and gamma-ray crosssections are destined to play a crucial role in reactors for space applications, for desalination in the agro-industrial complexes and for process heat. The problems are mainly those of neutron economy and materials damage another field for accelerators.

\section{Radiation processing}

Radiation processing may be used to increase the melting point, tensile strength, durability, and adhesive property of materials. Of the 270 accelerators in private industry, 46 are devoted to radiation processing on a production scale (exclusive of food processing). The current value of irradiated products, not including food, is about $\$ 200$ million per year, and much of this is due to electron accelerators. Radiation curing of coatings and finishes, especially for building materials, textiles and metals, is the area of greatest potential in the near future. Irradiation of plastics accounts for the largest share of capacity, with applications to packaging materials and electrical insulation showing great economic advantages and rapid commercial utilization.

As an example, pigmented monomers (without solvents) are processed by electron curing. The monomer is polymerized to produce a superior paint finish. The elimination of solvents from the paint industry should reduce the pollution problem.

\section{Accelerators in defence}

The role of accelerators in defence is not as great as it used to be, but it remains extremely important. Perhaps the most serious problem in this category is a book-keeping one. It has to do with detection, control and monitoring of fissionable materials, mainly those produced in power reactors. We must have the capability of nondestructive interrogation of materials. Accelerators need to be developed which produce neutrons and gamma rays of appropriate energy and intensity and which can be used to interrogate sealed packages to deduce their contents.

By 1980 , power reactors around the world will be producing plutonium at the rate of 200 pounds per day, sufficient for tens of nuclear weapons per day. Plutonium is now a commercial commodity, subject to private ownership. To monitor this situation simple, reliable methods must soon be developed for interrogating materials and accurately determining their makeup. Neutrons and gamma rays, produced by accelerators, offer one possibility and much has already been accomplished in this direction. Here, accelerators appear destined to play a central role for a long time to come.

Sooner or later there must evolve an all-inclusive international treaty for control of fissionable materials. Effective verification procedures are essential to the implementation of any agreement which involves production and distribution of fissionable materials and the limitation of development, production, and deployment of nuclear armaments. Highly specialized accelerators will certainly be part of the policing mechanism.

The nondestructive analysis techniques, particularly the acceleratorbased active interrogation techniques which give promise of high accuracy and sensitivity, may be immediately applicable to the identification and control of pollution in air and water. Neutron activation techniques, in particular, offer an extremely sensitive method of tracing low-level contaminants in air, water and soil.
Accelerators for medical purposes

Perhaps in no area has accelerator development had such a marked impact on mankind as in medicine in nuclear medicine and in radiation therapy. Radioisotopes are used in diagnostic medicine in connection with : thyroid uptake, blood volume determination, renal function, vitamin $\mathrm{B}_{12}$ absorption, fat malabsorption, RBC survival, iron turnover, cardiac output, hepatic function. At the moment most of the isotopes are discovered with accelerators and manufactured with reactors. However, accelerator-produced isotopes are increasing in volume.

of all isotope administrations, about one-third employ ${ }^{131} \mathrm{l}$. The use of ${ }^{123}$ I for in vivo thyroid uptake studies, brain scans, blood volume measurements, and liver and lung scans, reduces the radiation exposure to patients by about a factor of 100 over ${ }^{131} \mid$ because ${ }^{123}$ | has no particulate radiation and has a much shorter half-life. This reduced dose is especially important in pediatric and obstetric cases.

The second most widely used radioisotope in organic function studies is accelerator-produced ${ }^{57} \mathrm{Co}$ for vitamin $\mathrm{B}_{12}$ absorption tests. The reason is shorter half-life and greater counting efficiency for the lighter isotope.

The new Brookhaven linac and LAMPF can produce substantial amounts of ${ }^{72} \mathrm{Zn}$. Preliminary studies indicate this nuclide may become a routine scanning agent to be used in all males, over middle age, for early detection of prostatic cancer. No such agent exists at the present time although prostatic cancer is currently the third most frequent cause of death in male cancer patients.

In 1968, 300000 people were treated by radiotherapy, involving 3.5 million treatments, representing a 300000 dollar effort towards the arrest of cancer. 


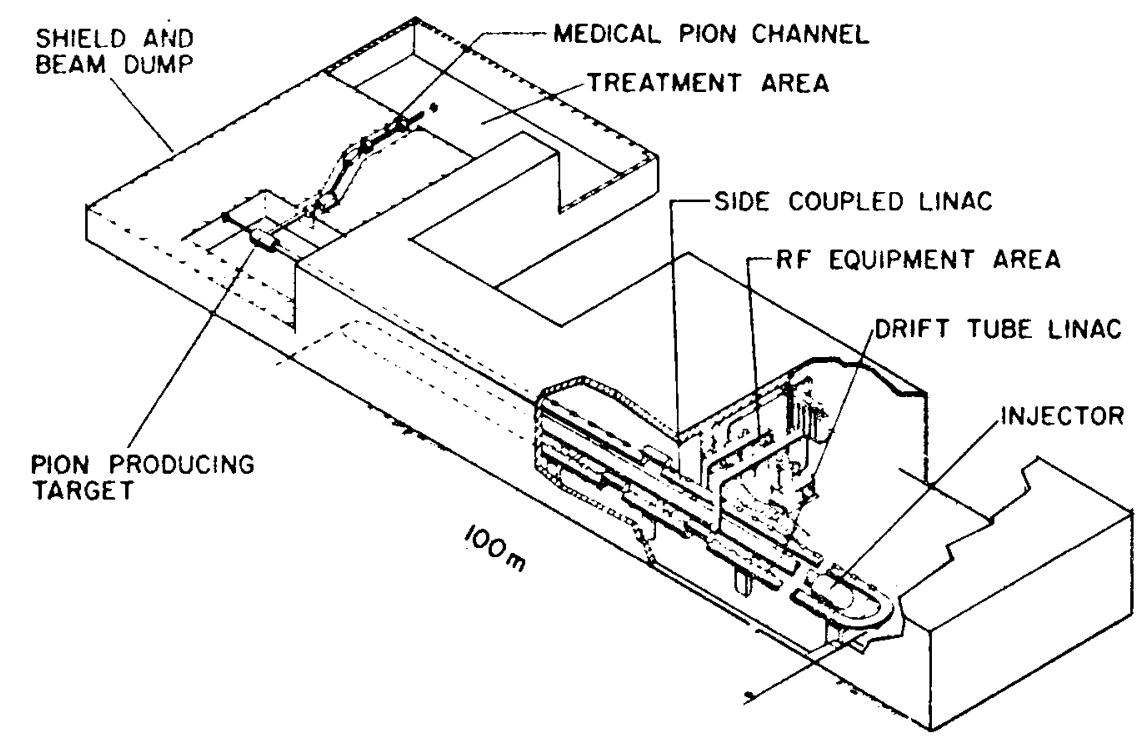

Concluding remarks

I have described what has been and what is now. But what about the future?

Some trends in accelerator applications are discernible. I have already mentioned that new accelerators need to be developed in order to achieve nuclear energy sources on the one hand and help with the world-wide management of fissionable materials on the other. New types of accelerators are needed for uses in every sphere from the preservation of food to the sterilization of sewage.

The field of medicine appears to have an insatiable appetite for accelerators which are tailored to their purposes. Just now, six cyclotrons for isotope production of the short-lived isotopes ${ }^{15} \mathrm{O},{ }^{13} \mathrm{~N}$ and ${ }^{11} \mathrm{C}(2,10$ and 20 minute half-lives) have been, or are in the process of being, installed in the USA.

With increased emphasis on nuclear medicine, the medical profession will be in a position to make use of new radionuclides which have properties more amenable to diagnostic procedures. Many of these can be provided by accelerators. Perhaps the most dramatic utilization of accelerators is in the treatment of malignancies where there are great advantages to be realized by using high-energy charged particles in radiation therapy. High-energy protons are much su- perior to $\mathrm{x}$ rays and we have been remiss in not using our high energy accelerators for this purpose. Our colleagues in the USSR are far ahead of us and I commend them for that.

In order to build LAMPF, a new accelerator structure had to be invented and developed. Very soon after, the feasibility, stability, and efficiency of this accelerator was demonstrated by building an electron prototype; the basic design features were adopted by industry, which is now producing them for $x$ ray machines of $4 \mathrm{MeV}$ (and higher) energy. At least five companies are building these machines; several dozen are a. ready installed in hospitals, several dozen more are under construction.

Lest you worry that higher energy machines be leit out of medical applications, let me assure you that this is not the case. The meson factories - LAMPF in USA, TRIUMF in Canada, and SIN in Switzerland are scheduled to provide negative pions for radiation therapy.

The problem of determining the beam energy necessary to achieve stopped pions uniformly and at a prescribed depth in the tumor volume seems near solution and a suitable pion channel has been designed.

It now appears that muons too may be useful in medicine - in diagnostic medicine. It occurred to me, several years ago, that muons might be used to determine elemental composition
An inexpensive meson factory, such as might be used exclusively for diagnostic and therapeutic medicine, as conceived by a Los Alamos group. A machine of this type could yield a $500 \mathrm{MeV}$. $0.5 \mathrm{~mA}$ proton beam for the production of intense beams of pions and muons.

in tissue just as neutron activation analysis is now used, but with less damage to the host organism. Recently some results have been obtained which are most encouraging.

The promise of pions and muons in medicine naturally raises the question of whether one might devise a very inexpensive, single-purpose meson factory. D. Nagle, E. Knapp, and $D$. Hagerman have given some thought to this question and have arrived at the concept shown in the drawing. A $3 \mathrm{MeV}$ pressurized Cockcroft-Walton feeds protons into a $400 \mathrm{MHz}$ drift-tube linac which in turn ejects into a $1200 \mathrm{MHz}$ side-coupled linac. The initial estimate is that a $500 \mathrm{MeV} 0.5 \mathrm{~mA}$ average current, lowduty factor machine can be built for about $\$ 5$ million. It is beginning to appear that two of the physicists most cherished particles are destined for a central role in diagnostic and therapeutic medicine.

I see particle accelerators assuming an ever more prominent role in our everyday life. It is not completely unreasonable to expect, within our life-time, the emergence of a mail order catalogue which would list :

1) Electron linacs (1-100 MeV) for the inspection and surveillance of nuclear materials and polymerization of plastics ;

2) Isochronous cyclotrons (100-400 $\mathrm{MeV}$ ) for isotope production and radiation therapy with protons and alpha particles ;

3) Meson factories for isotope production, radiation therapy with negative pions, and mu-activation analysis for medical diagnosis ;

4) Electrostatic machine (0-100 MeV) for radiation damage with neutrons and charged particles, isotope production, neutron crosssection measurements, and neutron activation analysis;

5), 6), 7), etc. And more to come which we have not yet thought out. 